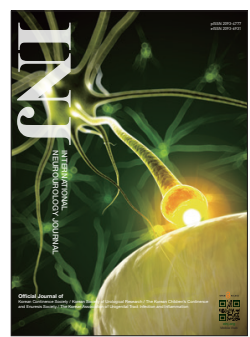

\title{
Do Lifestyle Factors Affect Lower Urinary Tract Symptoms? Results from the Korean Community Health Survey
}

\author{
Kyu Shik Kim ${ }^{1, \star}$, Jung Ki Jo ${ }^{1, *}$, Ji An Lee ${ }^{2,3}$, Bo Youl Choi ${ }^{2,3}$, Hong Sang Moon ${ }^{1, *}$ \\ ${ }^{1}$ Department of Urology, Hanyang University College of Medicine, Seoul, Korea \\ ${ }^{2}$ Institute for Health and Society, Hanyang University College of Medicine, Seoul, Korea \\ ${ }^{3}$ Department of Preventive Medicine, Hanyang University College of Medicine, Seoul, Korea
}

Purpose: To evaluate the relationships between lower urinary tract symptoms (LUTS) and lifestyle factors (physical activity, smoking, alcohol consumption, body mass index, and stress) in Korean men.

Methods: We analyzed the survey results of South Korean men $(n=64,439)$ who were 40 years of age or older among whom interviews were conducted using questionnaires. Trained interviewers performed face-to-face surveys using computer-assisted personal interviewing, the International Prostate Symptom Score (IPSS), and standard questions. We assessed the relationships of lifestyle factors (physical activity, cigarette smoking, alcohol intake, height, weight, and stress) with LUTS.

Results: We observed higher IPSS scores in participants who engaged in no exercise $(n=46,008$ [71.7\%], IPSS $=3.19 \pm 5.36)$ than in those who engaged in vigorous physical activity $(n=10,657$ [17.6\%], IPSS $=2.28 \pm 4.15)$. Former smokers showed higher total, storage, and voiding symptom IPSS scores than current smokers and nonsmokers. Nondrinkers had higher IPSS scores than current alcohol drinkers. In multivariable logistic regression analysis, we detected no relationship between LUTS and current alcohol drinking in the moderate and severe LUTS groups, using the mild LUTS group as a reference (moderate: $0.91 ; 95 \%$ confidence interval [CI], $0.91-0.91 ; \mathrm{P}<0.001$, severe: $0.78 ; 95 \% \mathrm{CI}, 0.78-0.78 ; \mathrm{P}<0.001$ ). Participants with moderate to severe stress showed higher total IPSS scores than those with no or mild stress ( $3.38 \pm 5.77$ vs. $2.88 \pm 4.90)$, with significant relationships between stress and LUTS found in the moderate and severe LUTS groups. In logistic regression analysis, stronger relationships were found for storage and voiding symptoms in the moderate and severe stress groups compared to the mild stress group.

Conclusions: A history of smoking, low levels of physical activity, low body mass index, and moderate to severe stress were associated with a greater severity of LUTS. Moderate to severe stress was also related to voiding symptoms. However, there was no association between alcohol intake and LUTS severity.

Keywords: Lower urinary tract symptoms; Life style; Tobacco smoking; Alcohols; Body mass index

- Research Ethics: This study was approved by the Institutional Review Board of the Hanyang University Hospital (2011-05CON-04-C).

- Conflict of Interest: HSM, an associate editor of the Editorial Board of International Neurourology Journal, is the corresponding author of this article. However, he played no role whatsoever in the editorial evaluation of this article or the decision to publish it. No potential conflict of interest relevant to this article was reported.

\section{INTRODUCTION}

Lower urinary tract symptoms (LUTS) occur in $20 \%-50 \%$ of people and diminish their quality of life $[1,2]$, particularly as they get older [2], leading to physical restrictions among the elderly [3]. LUTS in males are associated with various causes oth-

Corresponding author: Hong Sang Moon (iD https://orcid.org/0000-0003-2101-1019 Department of Urology, Hanyang University Guri Hospital, Hanyang University College of Medicine, 153 Gyeongchun-ro, Guri 11923, Korea

E-mail: moonuro@hanyang.ac.kr / Tel: +82-31-560-2374 / Fax: +82-31-560-2377

${ }^{*}$ Data Analysis Committee of the Korean Continence Society.

Submitted: January 10, 2019 / Accepted after revision: March 12, 2019 
er than prostatic diseases, such as water intake, diet, anticholinergic drugs, and bladder disorders [4]. However, when improvement is not observed, patients often restrict water consumption or search the internet to find ways to improve their symptoms in daily life. When such efforts do not succeed, patients present to physicians and undergo thorough work-ups, incurring high costs. In 2000, approximately 4.5 million patients received care in the United States with main complaints of LUTS. The total cost of such visits reached $\$ 1.1$ billion (USD) [5].

Epidemiological surveys have revealed that lifestyle factors such as food, exercise, alcohol intake, and smoking affect LUTS [6-9]. A previous study reported that physical activity was associated with LUTS and that appropriate amounts of alcohol improved LUTS by reducing inflammatory reactions and altering testosterone profiles $[10,11]$. Therefore, relevant lifestyle factors may affect sex-hormone metabolism; for example, alcohol consumption increases the metabolic clearance rate of testosterone, and inflammation caused by metabolic processes may be related to LUTS. However, not all previous research suggests that lifestyle-related factors are related to LUTS. For example, some studies have reported no relationship between LUTS and smoking $[11,12]$. In addition, while research on the relationships among sex, metabolic syndrome, overactive bladder (OAB), incontinence, and LUTS detected a correlation between female sex and incontinence, the correlations between OAB, LUTS, and male or female sex have not been clarified $[13,14]$. It is dif- ficult to investigate the exact association between daily lifestyle behaviors, such as smoking and alcohol intake, and $\mathrm{OAB}$ or LUTS, because it may be ethically improper to conduct randomized controlled trials to investigate certain lifestyle factors. Therefore, many cohort studies have been performed, with larger samples being more helpful for clarifying causality regarding LUTS.

In this study, the relationships of LUTS with the lifestyle factors of physical activity, smoking status, alcohol intake, body mass index (BMI), and stress - all of which have been identified as factors relevant to LUTS in the previous literature - were comparatively analyzed through a survey targeting a large sample of respondents residing in different regions of South Korea.

\section{MATERIALS AND METHODS}

\section{Study Group}

In this study, we analyzed raw data from the Korean Community Health Survey (KCHS) performed by the Korea Centers for Disease Control and Prevention from August to November 2011. This survey targeted South Koreans older than 19 years of age and was performed using 1:1 direct interview conducted through computer-assisted personal interviewing. The KCHS has been performed to generate regional health statistics since 2008.

At the nationwide level, the survey targeted a total of 229,226 respondents. It comprised a total of 247 question items in 18 ar-

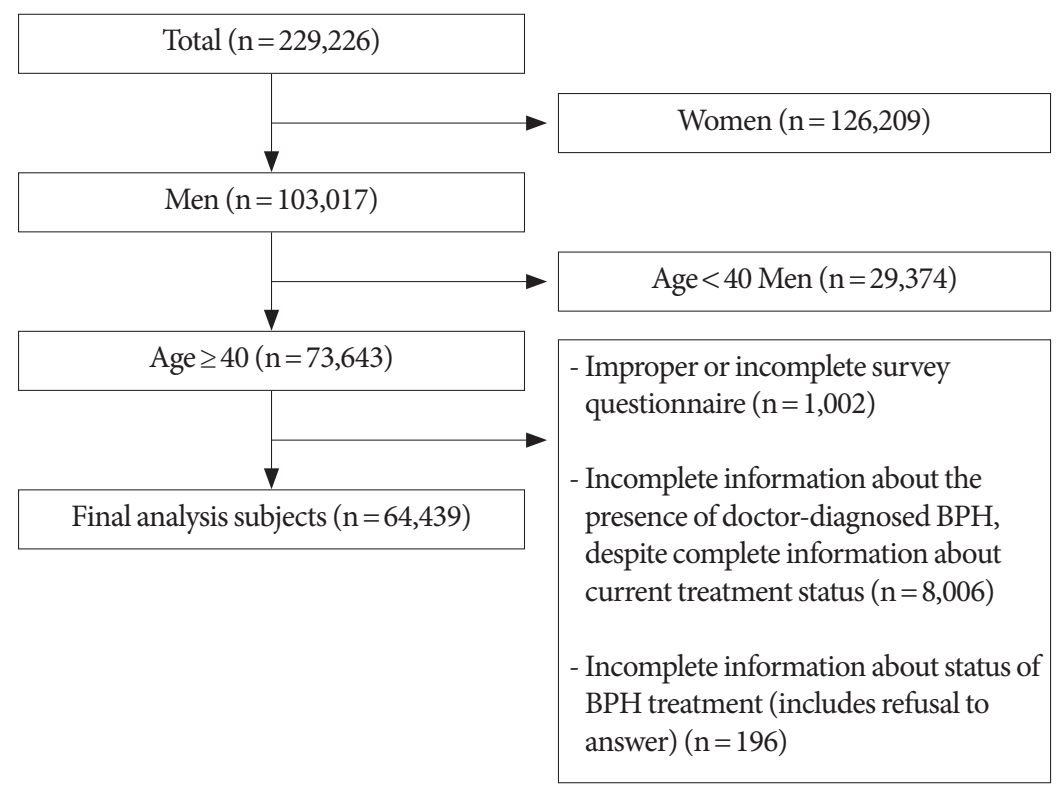

Fig. 1. Patient selection process. BPH, benign prostatic hyperplasia. 
eas, including a household survey, health conditions, preventive vaccinations, past history, the social environment, the physical environment, and education. Local community health surveys targeted 229,226 respondents, of whom 103,017 were men. Men aged over 40 years accounted for 73,643 of the respondents, of whom 1,002 were excluded because their question items were not complete (i.e., they refused to answer the questions or did not answer multiple questions); 8,006 because their questionnaires contained incomplete information on patients' status regarding doctor-diagnosed benign prostatic hyperplasia $(\mathrm{BPH})$, despite containing complete information on their current treatment status; and 196 because their questionnaires contained incomplete information on their BPH treatment (including those who refused to answer), leaving a final sample of 64,439 respondents (Fig. 1).

In this study, the questionnaire items related to International Prostate Symptom Score (IPSS), age, present smoking and drinking status, physical activity level, BMI, stress level, and chronic disease were used to analyze LUTS. We divided individuals into 3 groups according to LUTS: mild ( $8<$ IPSS), moderate $(8 \leq$ IPSS $\leq 19)$, and severe ( $20 \leq$ IPSS), based on the calculated total IPSS score, which included 7 related questionnaire items.

Regarding smoking status, participants who replied 'no' to the question of past smoking experience (having consumed over 5 packs of cigarettes) were classified as nonsmokers, and people who replied 'yes' (he or she reported still smoking daily or smoking sometimes) were classified as present smokers. People who smoked in the past but had since stopped were classified as former smokers.

Regarding drinking status, people who replied 'no' to the question of past drinking experience (having consumed more than 1 glass of liquor) were classified as nondrinkers. The category of nondrinkers also included persons who replied 'yes' to that question, but stated that they either did not drink during the past 1 year or drank less than once per month during the past 1 year. Persons who reported drinking more than once per month during the past 1 year were classified as current drinkers.

Physical activity was classified as moderate exercise (moderate physical activity over 5 times per week for more than 30 minutes per time during the most recent 1 week), vigorous exercise (vigorous physical activity over 3 times per week for more than 20 minutes per time during the most recent 1 week), and no exercise (neither moderate nor vigorous exercise). Height and body weight were surveyed by self-reporting, and participants were classified as underweight $(10 \leq \mathrm{BMI}[\mathrm{kg} /$ $\left.\left.\mathrm{m}^{2}\right]<18.5\right)$, normal weight $\left(18.5 \leq \mathrm{BMI}\left[\mathrm{kg} / \mathrm{m}^{2}\right]<25\right)$, or obese $\left(25 \leq\right.$ BMI $\left.\left[\mathrm{kg} / \mathrm{m}^{2}\right]<50\right)$.

Regarding stress-related question items, the questionnaire asked whether participants experienced stress in their daily life, and participants were asked to characterize their level of stress as none, moderate, or severe.

\section{Statistical Analysis}

In this study, we considered weighting and stratification variables, as well as cluster variables. As the raw data were collected using a complex sampling design, 64,439 individuals were included as the parent group so that information regarding sampling design would not be omitted. The Rao-Scott chi-square test was used in cross-analyses of categorical data. The relationships between voiding symptoms and smoking, drinking, BMI, physical activity, and stress were analyzed using survey logistic data based on a significance level of $5 \%$. At this point, diagnostic statuses of age and chronic diseases (hypertension, diabetes, dyslipidemia, stroke, myocardial infarction, angina pectoris, arthritis, osteoporosis, cataract, glaucoma, incontinence, $\mathrm{BPH}$, and depression) were corrected for in the analysis. We used SAS version 9.4 (SAS Institute, Cary, NC, USA) for statistical analyses.

\section{RESULTS}

Among a total of 103,017 male respondents, 73,643 were over 40 years of age. After excluding disqualified respondents, a total of 64,439 remained. Their average age was $56.75 \pm 10.65$ years and most did not exercise (71.1\%). However, among those who did engage in exercise, the percentage of men reporting vigorous exercise was higher than that of men reporting moderate exercise (16.6\% vs. $11.7 \%)$. There were fewer current smokers (42.0\%) than past smokers and nonsmokers together (58.0\%). There were more present drinkers than present nondrinkers, and most individuals reported a BMI in the range of 18.5-25.0 $\mathrm{kg} / \mathrm{m}^{2}$ (69.5\%). There were fewer individuals who felt severe stress than who felt no stress or slight stress (Table 1).

Mild LUTS was most common in respondents 40 to 49 years old, but in those over 70 years of age, the percentage of mild LUTS responses decreased to $63.0 \%$, while the percentage of severe LUTS responses increased to $8.2 \%$. Among those who engaged in vigorous exercise, LUTS were more likely to be mild than in the other groups (no exercise or moderate exercise) 
Table 1. Sociodemographic characteristics of the study population (Korean Community Health Survey, 2011) $(n=64,439)$

\begin{tabular}{|c|c|}
\hline Characteristic & Value \\
\hline $\begin{array}{l}\text { Age }(\mathrm{yr}) \\
40-49 \\
50-59 \\
60-69 \\
\geq 70\end{array}$ & $\begin{array}{l}56.75 \pm 10.65 \\
20,939(32.6) \\
19,098(29.6) \\
13,572(21.1) \\
10,830(16.8)\end{array}$ \\
\hline $\begin{array}{l}\text { Physical activity (exercise) } \\
\text { Severe } \\
\text { Moderate } \\
\text { None }\end{array}$ & $\begin{array}{r}\mathrm{n}=64,197 \\
10,657(16.6) \\
7,532(11.7) \\
46,008(71.7)\end{array}$ \\
\hline $\begin{array}{l}\text { Smoking } \\
\text { Current smoker } \\
\text { Former smoker } \\
\text { Nonsmoker }\end{array}$ & $\begin{array}{c}\mathrm{n}=64,433 \\
27,061(42.0) \\
24,421(38.0) \\
12,951(20.0)\end{array}$ \\
\hline $\begin{array}{l}\text { Alcohol intake } \\
\text { Current drinker } \\
\text { Nondrinker }\end{array}$ & $\begin{array}{c}\mathrm{n}=64,418 \\
44,338(68.8) \\
20,080(31.2)\end{array}$ \\
\hline $\begin{array}{l}\text { Body mass index }\left(\mathrm{kg} / \mathrm{m}^{2}\right) \\
10-18.5 \\
18.5-25.0 \\
25.0-50.0\end{array}$ & $\begin{array}{c}\mathrm{n}=62,845 \\
1,874(3.0) \\
43,689(69.5) \\
17,282(27.5)\end{array}$ \\
\hline $\begin{array}{l}\text { Stress (substantial or some stress, \%) } \\
\text { Moderate to severe } \\
\text { None to mild }\end{array}$ & $\begin{array}{c}\mathrm{n}=64,397 \\
15,589(24.2) \\
48,808(75.8)\end{array}$ \\
\hline $\begin{array}{l}\text { Chronic diseases }^{\mathrm{a})} \\
\text { Hypertension (yes, \%) } \\
\text { Diabetes (yes, \%) } \\
\text { Dyslipidemia (yes, \%) } \\
\text { Stroke (yes, \%) } \\
\text { Angina pectoris (yes, \%) } \\
\text { Myocardial infarction (yes, \%) } \\
\text { Osteoarthritis (yes, \%) } \\
\text { Osteoporosis (yes, \%) } \\
\text { Cataract (yes, \%) } \\
\text { Glaucoma (yes, \%) } \\
\text { Urinary incontinence (yes, \%) } \\
\text { Depression (yes, \%) }\end{array}$ & $\begin{array}{c}\mathrm{n}=47,473 \\
17,111(36.0) \\
7,317(15.4) \\
6,989(14.7) \\
1,657(3.5) \\
1,275(2.7) \\
1,298(2.7) \\
4,227(8.9) \\
824(1.7) \\
5,321(11.2) \\
623(1.3) \\
119(0.3) \\
712(1.5)\end{array}$ \\
\hline
\end{tabular}

Value are expressed as mean \pm standard deviation or number (\%).

${ }^{\text {a) }}$ Self-report of physician diagnosis.

(92.7\%) and in among those who reported severe LUTS, vigorous physical activity was proportionally less common (1.0\%) than no exercise or moderate exercise. In contrast, among those who did not engage in physical activity at all, mild LUTS were less common than in the other 2 groups (89.3\%) and severe LUTS were more common (1.8\%). The patient groups defined by LUTS severity and stratified by physical activity showed significant distributional differences $(\mathrm{P}<0.001)$. Former smokers showed a lower proportion than nonsmokers and current smok- ers in the mild LUTS group (87.0\%), but in the severe LUTS group, there was a higher proportion of former smokers $(2.0 \%)$ than of nonsmokers and current smokers. However, the highest proportion relative to LUTS status was found for the proportion of current smokers in the mild LUTS group (91.7\%). In the analyses of LUTS based on alcohol drinking status, current alcohol drinkers were more likely to have a low IPSS score than nondrinkers, and in an intergroup comparison based on stage of LUTS, current alcohol drinkers showed lower average IPSS scores than nondrinkers. In the underweight group, mild LUTS severity was less common than in the normal weight and overweight groups $(71.1 \%)$, and severe LUTS was more common than in the normal weight and overweight groups $(25.81 \pm 4.87$, 167 [7.8\%], $\mathrm{P}<0.001)$. When analyzing LUTS based on stress level, patients reporting severe stress showed higher average IPSS scores $(3.38 \pm 5.77$ vs. $2.88 \pm 4.90)$ than those who felt slight or no stress (Table 2).

Based on their survey responses, participants were classified and analyzed in terms of whether they did or did not have storage or voiding symptoms. As age increased, storage symptom scores increased in both participants who had no storage symptoms and those who did have storage symptoms. In terms of physical activity level, among those who did not exercise, storage scores were higher than among those who engaged in vigorous exercise ( $11.47 \pm 6.40$ vs. $12.84 \pm 7.26)$. As age increased, voiding symptom scores increased in both participants who had no voiding symptoms and those who did have voiding symptoms. In terms of physical activity levels, voiding scores were lower among those who engaged in vigorous exercise than among those who did not $(13.08 \pm 6.06$ vs. $14.33 \pm 6.86, \mathrm{P}<$ 0.001 ). In an analysis based on smoking status, the storage and voiding symptom scores were both highest among former smokers. In those with both storage symptoms and voiding symptoms, higher BMI was associated with a lower IPSS score. The storage symptom scores of individuals reporting severe stress were higher than those of their counterparts $(13.57 \pm 7.64$ vs. $12.16 \pm 6.92, \mathrm{P}<0.001)$, as were their voiding symptom scores ( $14.80 \pm 7.21$ vs. $13.82 \pm 6.52, \mathrm{P}<0.001)$ (Table 3).

We explored the relationships between lifestyle factors and LUTS using survey logistic regression. As respondents aged, their risk of moderate or severe LUTS increased. In particular, among individuals in their 60s or younger, the severity of LUTS symptoms tended to be mild or moderate, but among those over 70, the odds ratio [OR] for moderate LUTS was $7.24(95 \%$ confidence interval [CI], 7.24-7.24, $\mathrm{P}<0.001$ ) and that for se- 
Table 2. Selected characteristics of study subjects according to lower urinary tract symptoms (LUTS)

\begin{tabular}{|c|c|c|c|c|c|c|c|c|c|}
\hline \multirow{3}{*}{ Characteristic } & \multirow{2}{*}{\multicolumn{2}{|c|}{ Total $(\mathrm{n}=64,439)$}} & \multicolumn{6}{|c|}{ LUTS severity } & \multirow{3}{*}{ P-value } \\
\hline & & & \multicolumn{2}{|c|}{ Mild (8 > IPSS) } & \multicolumn{2}{|c|}{ Moderate $(8 \leq$ IPSS $\leq 19)$} & \multicolumn{2}{|c|}{ Severe $(20 \leq$ IPSS $)$} & \\
\hline & Score & No. (\%) & Score & No. $(\%)$ & Score & No. (\%) & Score & No. (\%) & \\
\hline No. of subjects & & $64,439(56.8)$ & & $56,295(55.2)$ & & $6,677(66.6)$ & & $1,467(70.8)$ & \\
\hline Age (yr) & & & & & & & & & $<0.001$ \\
\hline $40-49$ & $1.18 \pm 2.63$ & $20,939(32.5)$ & $0.84 \pm 1.48$ & $20,322(96.7)$ & $10.97 \pm 2.89$ & $548(2.9)$ & $25.26 \pm 4.20$ & $69(0.3)$ & \\
\hline $50-59$ & $2.02 \pm 3.68$ & $19,098(29.6)$ & $1.22 \pm 1.73$ & $17,774(92.7)$ & $11.14 \pm 3.03$ & $1,162(6.5)$ & $24.83 \pm 4.24$ & $162(0.8)$ & \\
\hline $60-69$ & $3.90 \pm 5.37$ & $13,572(21.1)$ & $1.91 \pm 2.07$ & $11,270(82.1)$ & $11.64 \pm 3.13$ & $1,962(15.2)$ & $25.29 \pm 4.38$ & $340(2.7)$ & \\
\hline$\geq 70$ & $7.13 \pm 7.56$ & $10,830(16.8)$ & $2.53 \pm 2.23$ & $6,929(63.0)$ & $12.20 \pm 3.27$ & $3,005(28.8)$ & $25.70 \pm 4.67$ & $896(8.2)$ & \\
\hline Physical activity (exercise) & & & & & & & & & $<0.001$ \\
\hline Severe & $2.28 \pm 4.15$ & $10,657(16.6)$ & $1.22 \pm 1.76$ & $9,721(92.7)$ & $11.58 \pm 3.13$ & $818(6.4)$ & $25.15 \pm 4.37$ & $118(1.0)$ & \\
\hline Moderate & $2.90 \pm 4.88$ & 7,532 (11.7) & $1.42 \pm 1.90$ & $6,644(89.7)$ & $11.68 \pm 3.15$ & $737(8.8)$ & $24.98 \pm 4.52$ & $151(1.5)$ & \\
\hline None & $3.19 \pm 5.36$ & $46,008(71.7)$ & $1.41 \pm 1.91$ & $39,723(89.3)$ & $11.79 \pm 3.21$ & $5,094(8.9)$ & $25.58 \pm 4.56$ & $1,191(1.8)$ & \\
\hline Smoking & & & & & & & & & $<0.001$ \\
\hline Nonsmoker & $2.75 \pm 4.83$ & $12,951(20.1)$ & $1.32 \pm 1.82$ & $11,491(91.1)$ & $11.71 \pm 3.13$ & $1,221(7.4)$ & $25.74 \pm 4.46$ & $239(1.5)$ & \\
\hline Former smoker & $3.69 \pm 5.65$ & $24,421(37.9)$ & $1.62 \pm 1.98$ & $20,432(87.0)$ & $11.81 \pm 3.25$ & $3,249(10.9)$ & $25.39 \pm 4.65$ & $740(2.0)$ & \\
\hline Current smoker & $2.50 \pm 4.69$ & $27,061(42.0)$ & $1.21 \pm 1.80$ & $24,366(91.7)$ & $11.69 \pm 3.14$ & $2,207(7.0)$ & $25.50 \pm 4.41$ & $488(1.3)$ & \\
\hline Alcohol intake & & & & & & & & & $<0.001$ \\
\hline Current drinker & $2.56 \pm 4.53$ & $44,338(68.9)$ & $1.56 \pm 2.00$ & $39,875(91.7)$ & $11.60 \pm 3.13$ & $3,758(7.2)$ & $25.12 \pm 4.23$ & $705(1.1)$ & \\
\hline Nondrinker & $3.99 \pm 6.15$ & $20,080(31.1)$ & $1.31 \pm 1.83$ & $16,400(84.8)$ & $11.95 \pm 3.25$ & $2,919(12.2)$ & $25.83 \pm 4.79$ & $761(3.0)$ & \\
\hline Body mass index $\left(\mathrm{kg} / \mathrm{m}^{2}\right)$ & & & & & & & & & $<0.001$ \\
\hline $10-18.5$ & $6.55 \pm 7.88$ & $1,874(3.0)$ & $2.06 \pm 2.19$ & $1,269(71.1)$ & $12.22 \pm 3.32$ & $438(21.1)$ & $25.81 \pm 4.87$ & $167(7.8)$ & \\
\hline $18.5-25.0$ & $2.97 \pm 5.05$ & $43,689(69.5)$ & $1.38 \pm 1.89$ & $38,198(89.7)$ & $11.74 \pm 3.20$ & $4,570(8.8)$ & $25.40 \pm 4.47$ & $921(1.6)$ & \\
\hline $25.0-50.0$ & $2.37 \pm 4.28$ & $17,282(27.5)$ & $1.28 \pm 1.80$ & $15,751(92.4)$ & $11.51 \pm 3.05$ & $1,294(6.4)$ & $25.10 \pm 4.28$ & $237(1.1)$ & \\
\hline Stress & & & & & & & & & $<0.001$ \\
\hline None to mild & $2.88 \pm 4.90$ & $48,808(89.7)$ & $1.38 \pm 1.87$ & $42,990(90.6)$ & $11.69 \pm 3.17$ & $4,860(8.1)$ & $25.29 \pm 4.49$ & $958(1.4)$ & \\
\hline Moderate to severe & $3.38 \pm 5.77$ & $5,589(10.3)$ & $1.37 \pm 1.92$ & $13,276(88.2)$ & $11.90 \pm 3.24$ & $1,808(9.5)$ & $25.86 \pm 4.61$ & $505(2.2)$ & \\
\hline
\end{tabular}

Value are expressed as mean \pm standard deviation unless otherwise indicated.

IPSS, International Prostate Symptom Score.

${ }^{a)}$ Rao-Scott chi-square.

vere LUTS was 11.73 (95\% CI, 11.73-11.73, P<0.001). Lower levels of exercise were associated with an increased risk of LUTS and a tendency for LUTS to be more severe, with an OR of 1.11 for moderate LUTS associated with moderate physical activity (95\% CI, 1.11-1.11; $\mathrm{P}<0.001)$ and an OR of 1.14 for no physical activity (OR, 1.14; 95\% CI, 1.14-1.14; $\mathrm{P}<0.001$ ). In both former and current smokers, the risk elevation for moderate LUTS was significant (former smokers: OR, 1.28; 95\% CI, $1.28-1.28$; $\mathrm{P}<0.001$, current smokers: OR, 1.18; 95\% CI, 1.181.18 ; $\mathrm{P}<0.001)$. Among those with severe LUTS, former smokers exhibited the same risk as nonsmokers, but the risk was higher among current smokers (current smokers: OR, 1.2; 95\%

\section{CI, 1.2-1.2; $\mathrm{P}<0.001)$.}

Current alcohol drinkers showed lower risk than nondrinkers for both moderate and severe LUTS. Similarly, for both moderate and severe LUTS, underweight individuals were at a higher risk than normal-weight individuals (moderate: OR, 1.26; 95\% CI, 1.26-1.26; $\mathrm{P}<0.001$, severe: OR, 1.91; 95\% CI, 1.91-1.91; $\mathrm{P}<0.001)$. However, among overweight individuals, the risk was lower. In other words, underweight individuals were at the highest risk for LUTS. Among those who reported feeling severe stress, the risk for moderate LUTS was elevated (OR, 1.59; 95\% CI, 1.59-1.59; $\mathrm{P}<0.001)$, and the same pattern was found for severe LUTS (OR, 1.87; 95\% CI, 1.87-1.87; $\mathrm{P}<0.001)$ (Table 4). 


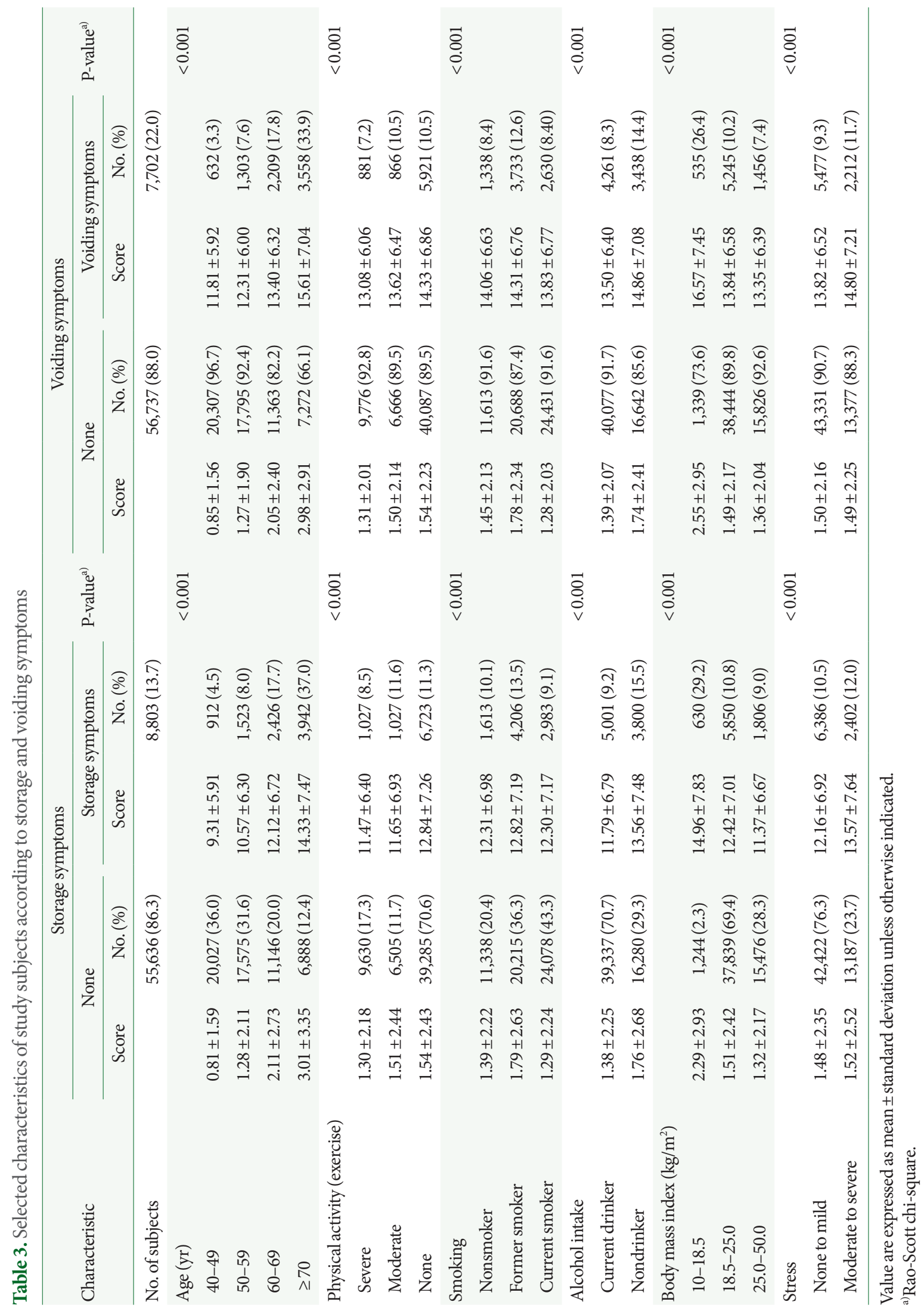


Table 4. Relationships between lifestyle factors and LUTS severity

\begin{tabular}{|c|c|c|c|c|c|c|}
\hline \multirow{3}{*}{ Variable } & \multicolumn{6}{|c|}{ Multivariable model } \\
\hline & \multicolumn{3}{|c|}{ Moderate $(8 \leq$ IPSS $\leq 19)$} & \multicolumn{3}{|c|}{ Severe $(20 \leq$ IPSS $)$} \\
\hline & OR & $95 \%$ CI & P-value & OR & $95 \%$ CI & P-value ${ }^{a}$ \\
\hline \multicolumn{7}{|l|}{ Age (yr) } \\
\hline $40-49$ & 1.00 & reference & - & 1.00 & reference & - \\
\hline $50-59$ & 2.09 & $2.09-2.09$ & $<0.001$ & 1.92 & $1.92-1.92$ & $<0.001$ \\
\hline $60-69$ & 4.97 & $4.97-4.97$ & $<0.001$ & 5.81 & $5.81-5.81$ & $<0.001$ \\
\hline$\geq 70$ & 7.24 & $7.24-7.24$ & $<0.001$ & 11.73 & $11.73-11.74$ & $<0.001$ \\
\hline \multicolumn{7}{|c|}{ Physical activity (exercise) } \\
\hline Severe & 1.00 & reference & - & 1.00 & reference & - \\
\hline Moderate & 1.11 & $1.11-1.11$ & $<0.001$ & 1.07 & $1.07-1.07$ & $<0.001$ \\
\hline None & 1.14 & $1.14-1.15$ & $<0.001$ & 1.17 & $1.17-1.17$ & $<0.001$ \\
\hline \multicolumn{7}{|l|}{ Smoking } \\
\hline Nonsmoker & 1.00 & reference & - & 1.00 & reference & - \\
\hline Former smoker & 1.28 & $1.28-1.28$ & $<0.001$ & 1.07 & $1.07-1.07$ & $<0.001$ \\
\hline Current smoker & 1.18 & $1.18-1.18$ & $<0.001$ & 1.2 & $1.2-1.2$ & $<0.001$ \\
\hline \multicolumn{7}{|l|}{ Alcohol intake } \\
\hline Current drinker & 1.00 & reference & - & 1.00 & reference & - \\
\hline Nondrinker & 0.91 & $0.91-0.91$ & $<0.001$ & 0.78 & $0.78-0.78$ & $<0.001$ \\
\hline \multicolumn{7}{|c|}{ Body mass index $\left(\mathrm{kg} / \mathrm{m}^{2}\right)$} \\
\hline $10-18.5$ & 1.00 & reference & - & 1.00 & reference & - \\
\hline $18.5-25.0$ & 1.26 & $1.26-1.26$ & & 1.91 & $1.91-1.92$ & \\
\hline $25.0-50.0$ & 0.8 & $0.8-0.8$ & & 0.88 & $0.88-0.88$ & \\
\hline \multicolumn{7}{|l|}{ Stress } \\
\hline None to mild & 1.00 & reference & - & 1.00 & reference & - \\
\hline Moderate to severe & 1.59 & $1.59-1.59$ & $<0.001$ & 1.87 & $1.87-1.87$ & $<0.001$ \\
\hline
\end{tabular}

IPSS, International Prostate Symptom Score; LUTS, lower urinary tract symptoms; OR, odds ratio; CI, confidence interval.

Multivariable model: adjusted for age, smoking status, drinking status, body mass index, stress, physical activity, and chronic diseases (hypertension, diabetes, dyslipidemia, stroke, angina pectoris, myocardial infarction, osteoarthritis, osteoporosis, cataract, glaucoma, depression).

a) $\mathrm{P}$-values and $\mathrm{P}$-values for trend were determined by multivariable survey logistic regression analyses.

Lower levels of physical activity were associated with higher risks for both storage and voiding symptoms. Compared with nonsmokers, former and current smokers experienced higher risks of storage and voiding symptoms. Present drinkers were at a lower risk for storage and voiding symptoms than nondrinkers (storage symptoms: current drinkers, OR, 0.89; 95\% CI, 0.830.96; $\mathrm{P}<0.001$; voiding symptoms: current drinkers, $\mathrm{OR}, 0.88$; $95 \% \mathrm{CI}, 0.81-0.95 ; \mathrm{P}<0.0001)$. Underweight was associated with an elevated risk for both storage and voiding symptoms (storage symptoms: [BMI 10-18.5 kg/m²], OR, 1.96; 95\% CI, 1.68-2.29; $\mathrm{P}<0.001$, [BMI 25.0-50.0 kg/m²] OR, 0.93; 95\% CI, 0.845-1; $\mathrm{P}<0.001$; voiding symptoms: [BMI $10-18.5 \mathrm{~kg} / \mathrm{m}^{2}$ ] OR, 1.73;
95\% CI, 1.46-2.06; P<0.001, [BMI 25.0-50.0 kg/m²] OR, 0.79; 95\% CI, 0.72-0.86; $\mathrm{P}<0.001)$. Participants experiencing moderate to severe stress were at a higher risk for both storage and voiding symptoms (storage symptoms: moderate to severe, OR. 1.52; 95\% CI, 1.4-1.65; $\mathrm{P}<0.001$, voiding symptoms: moderate to severe, OR, 1.77; 95\% CI, 1.63-1.92; $\mathrm{P}<0.001$ ) (Table 5).

\section{DISCUSSION}

In this study, we observed close correlations between LUTS severity and age. Physical activity was correlated with LUTS, and exhibited closer correlations with voiding symptoms than with 
Table 5. Relationships between lifestyle factors and LUTS (storage and voiding symptoms in the IPSS)

\begin{tabular}{|c|c|c|c|c|c|c|}
\hline \multirow{3}{*}{ Variable } & \multicolumn{6}{|c|}{ Multivariable model } \\
\hline & \multicolumn{3}{|c|}{ Storage symptoms } & \multicolumn{3}{|c|}{ Voiding symptoms } \\
\hline & OR & $95 \% \mathrm{CI}$ & P-value & OR & $95 \% \mathrm{CI}$ & P-value ${ }^{a)}$ \\
\hline \multicolumn{7}{|l|}{ Age (yr) } \\
\hline $40-49$ & 1.00 & Reference & - & 1.00 & Reference & - \\
\hline $50-59$ & 1.74 & $1.56-1.95$ & $<0.001$ & 2.28 & $2.02-2.58$ & $<0.001$ \\
\hline $60-69$ & 3.81 & $3.4-4.26$ & $<0.001$ & 5.36 & $4.72-6.08$ & $<0.001$ \\
\hline$\geq 70$ & 8.98 & $7.96-10.13$ & $<0.001$ & 10.72 & $9.38-12.25$ & $<0.001$ \\
\hline \multicolumn{7}{|c|}{ Physical activity (exercise) } \\
\hline Severe & 1.00 & Reference & - & 1.00 & Reference & - \\
\hline Moderate & 1.11 & $0.96-1.28$ & 0.178 & 1.17 & $1.01-1.36$ & 0.037 \\
\hline None & 1.09 & $0.98-1.21$ & 0.114 & 1.19 & $1.07-1.33$ & 0 \\
\hline \multicolumn{7}{|l|}{ Smoking } \\
\hline Nonsmoker & 1.00 & Reference & - & 1.00 & Reference & - \\
\hline Former smoker & 1.16 & $1.05-1.28$ & 0.003 & 1.2 & $1.2-1.47$ & $<0.001$ \\
\hline Current smoker & 1.12 & $1.01-1.24$ & 0.027 & 1.29 & $1.16-1.44$ & $<0.001$ \\
\hline \multicolumn{7}{|l|}{ Alcohol intake } \\
\hline Current drinker & 1.00 & Reference & - & 1.00 & Reference & - \\
\hline Nondrinker & 0.89 & $0.83-0.96$ & 0.002 & 0.88 & $0.81-0.95$ & 0.001 \\
\hline \multicolumn{7}{|c|}{ Body mass index $\left(\mathrm{kg} / \mathrm{m}^{2}\right)$} \\
\hline $10-18.5$ & 1.00 & Reference & - & 1.00 & Reference & - \\
\hline $18.5-25.0$ & 1.96 & $1.68-2.29$ & $<0.001$ & 1.73 & $1.46-2.06$ & $<0.001$ \\
\hline $25.0-50.0$ & 0.93 & $0.85-1$ & 0.062 & 0.79 & $0.72-0.86$ & $<0.001$ \\
\hline \multicolumn{7}{|l|}{ Stress } \\
\hline None to mild & 1.00 & Reference & - & 1.00 & Reference & - \\
\hline Moderate to severe & 1.52 & $1.4-1.65$ & $<0.001$ & 1.77 & $1.63-1.92$ & $<0.001$ \\
\hline
\end{tabular}

LUTS, lower urinary tract symptoms; IPSS, International Prostate Symptom Score; OR, odds ratio; CI, confidence interval.

Multivariable model: adjusted for age, smoking status, drinking status, body mass index, stress, physical activity, and chronic diseases (hypertension, diabetes, dyslipidemia, stroke, angina pectoris, myocardial infarction, osteoarthritis, osteoporosis, cataract, glaucoma, depression).

${ }^{\text {a) }} \mathrm{P}$-values and $\mathrm{P}$-values for trend were determined by multivariable survey logistic regression analyses.

storage symptoms. Former smokers and current smokers were more likely to report LUTS than nonsmokers, and the correlations of LUTS with voiding symptoms were much higher than those with storage symptoms. Alcohol drinking status and LUTS were not correlated. Underweight individuals were more likely than obese individuals to report LUTS. Stress was correlated with LUTS, and the correlation increased with severity of stress.

As men age, the incidence of LUTS increases and the related symptoms may become severe, which significantly affects their quality of life [15]. LUTS comprise chronic urologic symptoms associated with $\mathrm{BPH}$ and bladder, prostate, or urethra problems [16]. Establishing the risk factors associated with LUTS could help not only to prevent LUTS, but also to improve LUTS once they occur. In previous research, drinking and smoking were found to be correlated with LUTS, as well as educational and socioeconomic status [17]. Previous studies also showed that metabolic syndrome was closely related to the presence of LUTS [18].

Physical activity improves insulin sensitivity and leads to changes in the sympathetic nervous system response, depending on activity level [6]. In addition, activity is associated with cardiovascular stability, weight loss, normalization of lipid profiles, and stabilization of blood circulation. Physical activity may therefore affect proliferation of the prostate through hor- 
monal changes [19]. Several previous studies reported that physical activity reduces LUTS $[19,20]$. In addition, in a crosssectional meta-analysis, intense physical activity was observed to reduce the risks of BPH and LUTS by $25 \%$ compared with sedentary habits [21]. However, in most other research, physical activity status had a minimal relationship with improvement of LUTS among individuals in middle age or old age $[9,22,23]$. In the present study, lower levels of physical activity were found to be associated with stronger relationships with the risk for LUTS overall and for moderate LUTS. In addition, voiding symptoms were also closely related to minimal levels of physical activity. IPSS scores were higher in participants who engaged in no exercise than those who engaged in moderate or vigorous exercise. Logistic regression analysis also confirmed this relationship with physical activity.

Some previous research found no correlations between smoking status and LUTS; furthermore, there is no correlation between smoking and $\mathrm{BPH}$ [24] or the amount of nocturia in males [25]. However, nicotine increases sympathetic nervous system activity and the tone of smooth bladder muscle and may affect the storage system [26]. In addition, smoking causes imbalances between nutrition and hormones, and may affect collagen synthesis in the bladder [9]. In an analysis of the relationships between $\mathrm{OAB}$ and smoking, irritative symptoms were not involved, but obstructive symptoms were [9]. However, correlations between LUTS and smoking have been found in other studies. Smoking reduced the risk of decreased maximum flow rate force, although some studies have indicated that there were no correlations between such symptoms and smoking [27]. In addition, symptom status shows different correlations with smoking depending on sex; in particular, there is no special relationship with male sex [28]. In a prospective longitudinal cohort observed for 3 years, heavier smokers were more likely to experience storage symptoms [23]. In the present study, among males, past and present smoking affected LUTS, involving both storage and voiding symptoms. Voiding symptoms exhibited especially close correlations.

It was previously reported that moderate alcohol drinking reduced the risk of coronary artery disease and slowed the progress of BPH [8]. However, LUTS is correlated not only with prostate-related disease, but also with bladder-related disease. The bladder is both directly and indirectly affected by alcohol, diuretics, insulin sensitivity, and hormones [6]. Putative correlations between drinking and LUTS remain controversial, as for smoking. In prospective research performed for 3 years, there was no correlation between drinking (including heavy drinking [ $>40 \mathrm{~g} /$ day]) and LUTS [23]. In addition, in a recent metaanalysis, there was no correlation between drinking status and LUTS [8]. However, in other studies, heavy alcohol consumption was shown to affect incontinence and irritative LUTS [29]. In the present study, current alcohol drinking showed no association with moderate or severe LUTS, using mild LUTS as the reference group.

Obesity is related to insulin concentrations, and may lead to LUTS [30]. High concentrations of insulin may more readily lead to BPH and LUTS in obese men than in men of normal body weight by increasing sympathetic nerve activity and the activity of prostate smooth muscle [30]. However, in the EPICHeidelberg cohort study, no correlation was found between high BMI (including waist circumference) and LUTS during 10 years of follow-up. However, in those whose BMI increased for $10-14$ years, there was a $30 \%$ higher correlation with moderate/ severe LUTS [31]. In research carried out in Australia, increases in BMI were correlated with storage symptom progress [20], but in the Olmsted County Study, there was no correlation between increased IPSS or weight change and LUTS [32]. In prospective research carried out in Korea, no correlations were found between BMI or waist circumference and LUTS [23]. In the present study, we observed a relationship between underweight and moderate or severe LUTS, using mild LUTS as a reference group, whereas no such relationship was found for normal BMI. Among those whose BMI was high, there was no correlation.

Limited research has addressed the putative correlations between LUTS and stress. The detailed mechanism underlying this relationship has not been adequately researched, but both emotional stress and environmental stress are correlated with LUTS. LUTS is related to uneasiness and depression, which in turn are correlated with environmental factors, psychiatric factors, and hereditary factors [33]. Emotional stress affects the bladder due to relationships among hypothalamic-pituitary axis activity, dysregulation of serotonergic pathways, and central sensitization [34]. In a study of the relationship between occupational stress and LUTS, OAB assessments performed by female nurses confirmed the presence of correlations among such factors [35]. In the present study, we compared mild, moderate, and severe LUTS groups by stress level, and found a correlation between moderate to severe stress and storage and voiding symptoms. This study was a questionnaire-based cross-sectional investigation, and did not assess specific lifestyle changes over 
time. Therefore, future longitudinal investigations assessing LUTS improvement after lifestyle changes are necessary.

In conclusion, a history of smoking, low physical activity, low BMI, and moderate to severe stress were associated with LUTS severity. Moderate to severe stress was associated with voiding symptoms. However, there was no association between alcohol intake and LUTS severity.

\section{AUTHOR CONTRIBUTION STATEMENT}

- Full access to all the data in the study and takes responsibility for the integrity of the data and the accuracy of the data analysis: $K S K, J A L$

- Study concept and design: HSM, BYC

- Acquisition of data: JAL

- Analysis and interpretation of data: KSK

- Drafting of the manuscript: KSK

-Critical revision of the manuscript for important intellectual content: KSK, HSM

- Statistical analysis: $J A L$

- Obtained funding: None

- Administrative, technical, or material support: HSM, JAL, BYC

- Study supervision: HSM

\section{REFERENCES}

1. Coyne KS, Sexton CC, Thompson CL, Milsom I, Irwin D, Kopp ZS, et al. The prevalence of lower urinary tract symptoms (LUTS) in the USA, the UK and Sweden: results from the Epidemiology of LUTS (EpiLUTS) study. BJU Int 2009;104:352-60.

2. Coyne KS, Wein AJ, Tubaro A, Sexton CC, Thompson CL, Kopp ZS, et al. The burden of lower urinary tract symptoms: evaluating the effect of LUTS on health-related quality of life, anxiety and depression: EpiLUTS. BJU Int 2009;103 Suppl 3:4-11.

3. Boyle P, Robertson C, Mazzetta C, Keech M, Hobbs FD, Fourcade $\mathrm{R}$, et al. The prevalence of lower urinary tract symptoms in men and women in four centres. The UrEpik study. BJU Int 2003;92: 409-14.

4. Su L, Guess HA, Girman CJ, Jacobsen SJ, Oesterling JE, Panser LA, et al. Adverse effects of medications on urinary symptoms and flow rate: a community-based study. J Clin Epidemiol 1996;49:483-7.

5. Wei JT, Calhoun E, Jacobsen SJ. Urologic diseases in america project: benign prostatic hyperplasia. J Urol 2008;179(5 Suppl):S75-80.

6. Rohrmann S, Crespo CJ, Weber JR, Smit E, Giovannucci E, Platz EA. Association of cigarette smoking, alcohol consumption and physical activity with lower urinary tract symptoms in older American men: findings from the third National Health And Nutrition Examination Survey. BJU Int 2005;96:77-82.

7. Lee HW, Kim SA, Nam JW, Kim MK, Choi BY, Moon HS. The study about physical activity for subjects with prevention of benign prostate hyperplasia. Int Neurourol J 2014;18:155-62.

8. Parsons JK, Im R. Alcohol consumption is associated with a decreased risk of benign prostatic hyperplasia. J Urol 2009;182:14638.

9. Maserejian NN, Kupelian V, Miyasato G, McVary KT, McKinlay JB. Are physical activity, smoking and alcohol consumption associated with lower urinary tract symptoms in men or women? Results from a population based observational study. J Urol 2012;188:4905.

10. Brown JS, Wing R, Barrett-Connor E, Nyberg LM, Kusek JW, Orchard TJ, et al. Lifestyle intervention is associated with lower prevalence of urinary incontinence: the Diabetes Prevention Program. Diabetes Care 2006;29:385-90.

11. Gordon GG, Altman K, Southren AL, Rubin E, Lieber CS. Effect of alcohol (ethanol) administration on sex-hormone metabolism in normal men. N Engl J Med 1976;295:793-7.

12. Araki H, Watanabe H, Mishina T, Nakao M. High-risk group for benign prostatic hypertrophy. Prostate 1983;4:253-64.

13. Bravi F, Bosetti C, Dal Maso L, Talamini R, Montella M, Negri E, et al. Food groups and risk of benign prostatic hyperplasia. Urology 2006;67:73-9.

14. Mommsen S, Foldspang A. Body mass index and adult female urinary incontinence. World J Urol 1994;12:319-22.

15. Bunn F, Kirby M, Pinkney E, Cardozo L, Chapple C, Chester K, et al. Is there a link between overactive bladder and the metabolic syndrome in women? A systematic review of observational studies. Int J Clin Pract 2015;69:199-217.

16. Madersbacher S, Alivizatos G, Nordling J, Sanz CR, Emberton M, de la Rosette JJ. EAU 2004 guidelines on assessment, therapy and follow-up of men with lower urinary tract symptoms suggestive of benign prostatic obstruction (BPH guidelines). Eur Urol 2004; 46:547-54.

17. Cornu JN, Lukacs B. Lower urinary tract symptoms in men: refocus on your patients. Eur Urol 2015;67:1110-1.

18. Haidinger G, Temml C, Schatzl G, Brössner C, Roehlich M, Schmidbauer CP, et al. Risk factors for lower urinary tract symptoms in elderly men. For the Prostate Study Group of the Austrian Society of Urology. Eur Urol 2000;37:413-20.

19. Pashootan P, Ploussard G, Cocaul A, de Gouvello A, Desgrandchamps F. Association between metabolic syndrome and severity 
of lower urinary tract symptoms (LUTS): an observational study in a 4666 European men cohort. BJU Int 2015;116:124-30.

20. Parsons JK, Messer K, White M, Barrett-Connor E, Bauer DC, Marshall LM, et al. Obesity increases and physical activity decreases lower urinary tract symptom risk in older men: the Osteoporotic Fractures in Men study. Eur Urol 2011;60:1173-80.

21. Martin S, Lange K, Haren MT, Taylor AW, Wittert G; Members of the Florey Adelaide Male Ageing Study. Risk factors for progression or improvement of lower urinary tract symptoms in a prospective cohort of men. J Urol 2014;191:130-7.

22. Parsons JK, Kashefi C. Physical activity, benign prostatic hyperplasia, and lower urinary tract symptoms. Eur Urol 2008;53:1228-35.

23. Marshall LM, Holton KF, Parsons JK, Lapidus JA, Ramsey K, Barrett-Connor E, et al. Lifestyle and health factors associated with progressing and remitting trajectories of untreated lower urinary tract symptoms among elderly men. Prostate Cancer Prostatic Dis 2014;17:265-72.

24. Choo MS, Han JH, Shin TY, Ko K, Lee WK, Cho ST, et al. Alcohol, smoking, physical activity, protein, and lower urinary tract symptoms: prospective longitudinal cohort. Int Neurourol J 2015;19:197206.

25. Roberts RO, Tsukamoto T, Kumamoto Y, Rhodes T, Masumori N, Miyake $\mathrm{H}$, et al. Association between cigarette smoking and prostatism in a Japanese community. Prostate 1997;30:154-9.

26. Kang D, Andriole GL, Van De Vooren RC, Crawford D, Chia D, Urban DA, et al. Risk behaviours and benign prostatic hyperplasia. BJU Int 2004;93:1241-5.
27. Narkiewicz K, van de Borne PJ, Hausberg M, Cooley RL, Winniford MD, Davison DE, et al. Cigarette smoking increases sympathetic outflow in humans. Circulation 1998;98:528-34.

28. Roberts RO, Jacobsen SJ, Rhodes T, Guess HA, Girman CJ, Panser LA, et al. Cigarette smoking and prostatism: a biphasic association? Urology 1994;43:797-801.

29. Rohrmann S, Fallin MD, Page WF, Reed T, Partin AW, Walsh PC, et al. Concordance rates and modifiable risk factors for lower urinary tract symptoms in twins. Epidemiology 2006;17:419-27.

30. Joseph MA, Harlow SD, Wei JT, Sarma AV, Dunn RL, Taylor JM, et al. Risk factors for lower urinary tract symptoms in a populationbased sample of African-American men. Am J Epidemiol 2003; 157:906-14

31. Hammarsten J, Högstedt B. Hyperinsulinaemia as a risk factor for developing benign prostatic hyperplasia. Eur Urol 2001;39:151-8.

32. Rohrmann S, Katzke VA, Kaaks R. Lifestyle and progression of lower urinary tract symptoms in German men-results from the EPIC-Heidelberg Cohort. Urology 2018;120:192-6.

33. St Sauver JL, Sarma AV, Hollingsworth JM, Jacobson DJ, McGree ME, Dunn RL, et al. Associations between modest weight changes and onset and progression of lower urinary tract symptoms in two population-based cohorts. Urology 2011;78:437-41.

34. Afari N, Gasperi M, Forsberg CW, Goldberg J, Buchwald D, Krieger JN. Heritability of lower urinary tract symptoms in men: a twin study. J Urol 2016;196:1486-92.

35. de Groat WC. Influence of central serotonergic mechanisms on lower urinary tract function. Urology 2002;59(5 Suppl 1):30-6. 